
https://doi.org/10.30534/ijns/2019/05832019

\title{
Patient Monitoring System Application Using Machine Learning
}

\author{
Shifana Sherin, Sahila Serin CH, Asma Blaisy KK \\ Student, India,shifanasherin2015@gmail.com \\ Student, India, shahalasharinch@ gmail.com \\ Student, India,asmablaisykk@gmail.com
}

\begin{abstract}
Generally in critical case-patients are supposed to be monitored continuously for their physical information as well as their health conditions. In the earlier methods the history of patients cannot be displayed, also bystanders are eagerly waiting long periods to know the condition of patients who are in critical situation. In the current paper, we tend to square measure employing a novel plan for continuous observance patients health condition. The healthcare scheme is focus on the measurement and monitoring various biological parameters of patients body like heart rate, oxygen saturation level in blood and temperature using a wireless body sensor and Android application and also after discharging the health status of the patients can be determined by the doctors through this application this help the patient to be alert. Doctors will access the data whenever required from any place and want not physically gifted.
\end{abstract}

Key words : Machine Learning, Clinical Decision Support Systems, Tarchanoff, Electrodermal.

\section{INTRODUCTION}

The ubiquitous patient monitoring system, it provides better smart alert health care application to people at anytime and anywhere in the world in a more economic and patient friendly manner. We thus need to collect the relevant data for the study of patient, then train the data as per the proposed algorithm of machine learning and thus predict the patient's possibility of curing diseases. On the input side ie, the data of the patients are entered into the application by easily available sensors in watches and measures the parameters of the patients. This information from the sensors are then transferred to For example, in the case of ICU, the nurses or other caretakers may not be available for constant monitoring of patient's health. Due to this, the patient's health becomes in a critical condition. So this continuous monitoring for a person is not possible by the current situation. The goal of this system is to monitor the patient's blood pressure, heartbeat rate, $\mathrm{SpO} 2$ etc. through a watch structured sensor and show the output of the patient parameters in the application[2]. The need for this patient monitoring system is in situations where the patient is unstable, physiological regulatory systems, in life-threatening conditions, critical physiological state. The main aim of patient monitoring is to give warning of early or dangerous diseases by training the system using machine learning. Monitoring of Patient's Blood Pressure, Heartbeat rate, body temperature, body position constantly is difficult in ICU's of a hospital. Existing Monitoring systems are wearable wireless and remote monitoring systems. Sensors are used to read the physiological parameters of the patient body. These sensors value directly measure by any controller or processor. In this methodology, the patient's vital signs like ECG, heart rate, breathing rate, temperature, $\mathrm{SpO} 2$ are captured and the values are entered into the database $[8,9,10]$. It is then uploaded into the net based mostly server and sent to the doctor's phone victimization robot technology. It conjointly permits the doctors to instantly remand their feedback to the nurse station.

The rest of the paper is organized as follows. Section 2 includes the detailed proposed system. Section 3 gives the details about the software used. Section 4 presents the design motivations through analysis of related work from the literature survey. Section 5 provides the result of the project and finally section 6 concludes the paper.

\subsection{Figure}

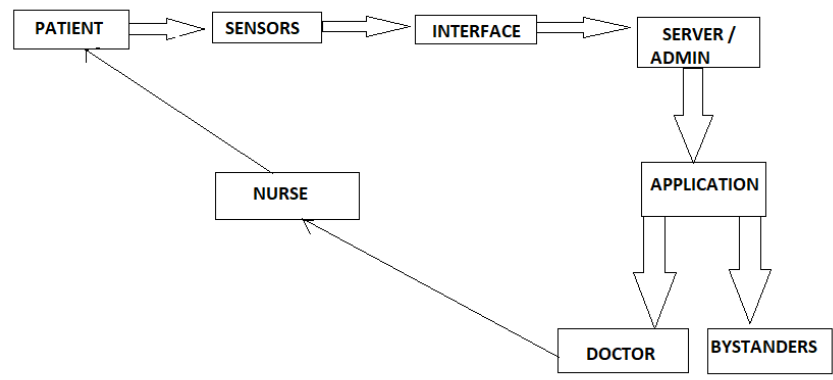

Figure 1: Over all process in a flow chart.

\section{REFERENCES}

A Health decision support system for disease diagnosis based on wearable medical sensors [1]. This paper introduce a hierarchical health decision support system for disease 
diagnosis that integrates health data from WMSs (wearable medical sensors) into CDSSs (clinical decision support systems).The proposed system has a multi-tier structure, starting with a WMS tier, backed by robust machine learning. It enables diseases to be tracked individually by a disease diagnosis module .It demonstrate the feasibility of a system through six disease diagnosis modules aimed at four ICD-10CM disease categories. Just the WMS tier offers spectacular diagnostic accuracies for diverse diseases like cardiovascular disease (86\%), type-2 polygenic disease (78\%), vesica disorder (99\%), bodily cavity kidney disease (94\%), and hypothyroid (95), renal pelvis nephritis (94\%), and hypothyroid (95).CDSSs have very limited access to patient's health status when he/she leaves the clinic .This results ,several deficiencies faced by today's CDSS. Patients cannot always remember all the previous disease symptoms .So this may be the only information source for physicians and for making decisions .WMSs sense physiological signals passively and continuously. Useful health inferences can be derived from these signals. These sensors form a powerful human machine interface for tracking the health condition of the user .Most current WMSs utilize a simple multi-threshold method to send alerts whenever signals fall outside the specified range.

Android based health care monitoring system [4]. Generally in critical case patients are supposed to be monitored continuously case the history of the patient cannot be displayed, only current data is displayed .In this paper, we are using a novel idea for continuous monitoring patient's health conditions. The health care scheme is focus on the measurement and Monitoring various biological parameters of patient's body like heart rate, oxygen saturation level in blood and temperature using a web server and android application .Doctor can continuously monitor the patient's condition on his smart phone using an Android application .Also the patient history will be stored on the web server and doctor can access the information whenever needed from anywhere and need not physically present.

Patient monitoring system using android technology[11]. Telemedicine is a rapidly developing application of clinic medicine where medical information is transferred through the phone or internet or other networks for the purpose of consulting and performing remote medical procedures or examinations In this technique, the patient's vital signs like $\mathrm{ECG}$, heart rate, breathing rate, temperature, $\mathrm{SpO} 2$ are captured and the values are entered into the database .It is then uploaded into the net based mostly server and sent to the doctor's phone victimization golem technology. It also enables the doctors to instantly send back their feedback to the nurse station. Prediction of health disease using machine learning $[5,6,12]$. With the increase in the heart stroke rates at juvenile ages, we need to put a system in place to be able to detect the symptoms of a heart stroke at Associate in Nursing early stage and therefore stop it. It is impractical for a common man to frequently undergo costly tests like the ECG and thus there needs to be a system in place which is handy and at the same time reliable, in predicting the chances of a heart disease .Thus proposed to develop an application which can predict the vulnerability of a heart disease given basic symptoms like age, sex, pulse rate etc. The machine learning algorithm neural networks has proven to be the most accurate and reliable algorithm and hence used in the proposed system

An economical Feature choice technique for classification in Health care Systems exploitation Machine Learning Techniques [3].Data mining may be used for an outsized quantity of applications .Among one is the health care systems .Usually, medical databases have giant quantities of knowledge concerning patients and their medical record .Analyzing this voluminous data manually is impossible .But this medical data contain very useful and valuable information which may save many lives if analyzed and utilized properly. Data mining technology is very effective for Health Care applications for identifying patterns and deriving useful information from these databases .Diabetes is one amongst the main causes of premature unhealthiness and death worldwide .In developing countries, less than half of people with diabetes are diagnosed.Without timely diagnoses and adequate treatment, complications and morbidity from diabetes rise exponentially .India has the world's largest polygenic disorder population, followed by China with 43.2 million .This paper describes concerning the applying of knowledge mining techniques for the detection of polygenic disorder in PIMA Indian polygenic disorder Dataset (PIDD).In this paper we tend to propose a Feature choice approach employing a combination of Ranker Search technique. The classification accuracy of eighty one resulted from our approach proves to be higher compared with previous results.

\section{ABBREVIATIONS}

$\begin{array}{ll}\text { CDSS } & \text { Clinical decision support systems } \\ \text { WMS } & \text { Wearable medical sensors } \\ \text { PIDD } & \text { PIMA Indian polygenic disorder Dataset } \\ \text { ECG } & \text { Electroencephalogram } \\ \text { SpO2 } & \text { Oxygen saturation } \\ \text { MLA } & \text { Machine Learning Algorithms } \\ \text { HDSS } & \text { Health decision support system }\end{array}$

\section{CONCLUSION}

This paper presents a medical terminology assignment scheme to know the patient's condition without communication with the doctor. It represented with three 
stages censoring stage (data collecting stage), storing stage and presenting stage. Patient's data is collected in censoring stage, the data is then moved to the database and stored there. When it needed a bystander or doctor can access it through the Android app by using patient's username and password. The system has many advantages first one is reduced time, the app will need only a few minutes for your requested data, also it is cost effective.

The main advantage is the use of machines can be reduced using these sensors and app. When the status of the patient is changed the app will update it instantly[7], also it will give alert messages in critical situations, so bystanders can take actions immediately. This whole process of our approach is unsupervised and hold the potential to handle large data.

\section{ACKNOWLEDGEMENT}

We would remember with grateful appreciation, the encouragement and support rendered by the Principal Dr. B Priestly Shan of Eranad Knowledge City Manjeri.

We express our deepest sense of gratitude to Head of the Department Mr.Adharsh T.K and our internal guide Ms.Naeema Mohamed Kutty, Designation in Computer Science and Engineering for their valuable advice and guidance.

We would always oblige for the helping hands of all other staff members of the department and all our friends and well-wishers, who directly or indirectly contributed in this venture.

Last but not least, we are indebted to God Almighty for being the guiding light throughout this project and helped us to complete the same within the stipulated time.

\section{REFERENCES}

1. Niraj K, Hongxu Yin "A Health Decision Support System for Disease Diagnosis based on Wearable Medical Sensors" Citation information: DOI 10.1109/TMSCS.2017.2710194, IEEE

2. K. Subramanya , Vishnuprasada V. Bhat, and Sandeep Kamath. A wearable device for monitoring galvanic skin response to accurately predict changes in blood pressure indexes and cardiovascular dynamics. 2013 Annual IEEE India Conference (INDICON). https://doi.org/10.1109/INDCON.2013.6726085

3. K Selvakuberan, D Kayathiri, B Harini, Dr M Indra Devi "An efficient Feature selection method for classification in Health care Systems using Machine Learning Techniques". 978-1-4244-8679-3/11/\$26.00 @2011. https://doi.org/10.1109/ICECTECH.2011.5941891

4. Maradugu Anil Kumar, Y.Ravi Sekhar. "Android Based Health Care Monitoring system”. 978-1- 4799- 6818- 3 /15/\$31.00 @ 2015 IEEE

5. Min Chen, Yixue Hao, Kai Hwang, Fellow, IEEE, Lu Wang, and Lin Wang. "Disease Prediction by Machine
Learning over Big data from Healthcare Communities" 2169-3536 (c) 2016 IEEE.

6. Vineet Kumar Singh, 2Saksham Bhatia, 3Ayush Verma, 4Shubhi Shaily,"Human Motion Prediction Using Machine Learning and Signal Processing". 978-1-5090-5013-0/17/\$31.00 @2017 IEEE.

7. Moses.E,2Melwin.P,3Vigneshwari," Instant Answering for Health Care system by Machine Learning Approach"978-1-5090-1277-0/16/\$31.00 @ 2016 IEEE.

8. Muhammad Aurangzeb Ahmad, Carly Eckert, Ankur Teredesai "interpretable machine learning in health care"2575-2634/18/\$31.00@ 2018IEEE.

9. Tanner Christensen, Abraham Frandsen, Seth Glazier, Jeffrey Humpherys David Kartchner" Machine Learning Methods for Disease Prediction with Claims data". 2575-2634/18/\$31.00 @2018 IEEE https://doi.org/10.1109/ICHI.2018.00108

10. Vahram Mouradian Armen Poghosyan, Levon Hovhannisyan" noninvasive Continuos mobile blood pressure monitoring using novel ppg optical Sensor" 978-1-4799-5511-4/15/\$31.00 @ 2015 IEEE

11. Prema Sundaram," PATIENT MONITORING SYSTEM USING ANDROID TECHNOLOGY" ( ) 2013, IJCSMC All Rights.

12. Aditi Gavhane, Gouthami Kokkula, Isha Pandya,Prof. Kailas Devadkar "prediction of Heart Disease Using Machine Learning” IEEE Xplore ISBN: 978 - 1- 53860965-1 